\title{
Alteration of Thiol-Disulfide Homeostasis in Fibromyalgia Syndrome
}

\author{
Ayca Tuzcuํㄹ Rabia Aydogan Baykara², Murat Alışık², Ahmet Omma4, Gunseli Karaca Acet², \\ Erdal Dogan², Medine Cumhur Cure ${ }^{5}$, Fatih Duygun ${ }^{6}$, Erkan Cure ${ }^{7, *}$, Ozcan Erel $^{8}$
}

\begin{abstract}
Background: Fibromyalgia syndrome (FMS) is an extra-articular rheumatological disease, characterized by widespread pain and somatic symptoms. The etiology has not yet been clarified. Oxidative stress may play an important role in FMS etiology. Thiol group is a very strong antioxidant. We aimed to investigate whether thiol/disulfide homeostasis in FMS is altered or not.

Material and methods: A total of 80 female FMS patients and 64 healthy female control individuals were included in this study. Thiol and disulfide values were measured by Erel's novel methods.

Results: Native thiol $(330.6 \pm 46.1$ vs. $356.8 \pm 55.5 \mu \mathrm{mol} / \mathrm{L}, \mathrm{p}=0.005)$ and native thiol/total thiol $(89.4 \pm 3.2 \mathrm{vs} .93 .3 \pm 4.0, \mathrm{p}<0.001)$ levels of FMS patients were significantly lower when compared to the values of control group. However, disulfide $(19.4 \pm 6.3 \mathrm{vs} .12 .2 \pm 6.3 \mu \mathrm{mol} / \mathrm{L}$, $p<0.001$ ) levels of FMS patients were significantly higher than healthy individuals. A negative correlation was found between the native thiol/total thiol and fibromyalgia impact questionnaire (FIQ) score among the FMS patients. A positive correlation was found between disulfide values and FIQ score among the patients.

Conclusions: In FMS patients, there was a significant correlation between the decrease in the thiol levels and an increase in the disulfide levels with the FIQ scores. We determined that thiol-disulfide rate was deteriorated in FMS patients and it increases in favor of disulfide amounts.
\end{abstract}

\section{KEYWORDS}

fibromyalgia; thiol; disulfide; thiol/disulfide homeostasis; oxidative stress

\section{AUTHOR AFFILIATIONS}

${ }^{1}$ Department of Biochemistry, Malatya Education and Research Hospital, Malatya, Turkey

2 Department of Physical Medicine and Rehabilitation, Malatya Education and Research Hospital, Malatya, Turkey

${ }^{3}$ Department of Clinical Biochemistry, Ankara Education and Research Hospital, Ankara, Turkey

${ }^{4}$ Division of Rheumatology, Department of Internal Medicine, Numune Education and Research Hospital, Ankara, Turkey

${ }^{5}$ Department of Biochemistry, Istanbul Laboratory, Istanbul, Turkey

${ }^{6}$ Departments of Orthopedics and Traumatology, Antalya Training and Research Hospital, Antalya, Turkey

7 Department of Internal Medicine, Camlica Erdem Hastanesi, Istanbul, Turkey

8 Department of Clinical Biochemistry, Faculty of Medicine, Yildirim Beyazit University, Ankara, Turkey

* Corresponding author: Department of Internal Medicine, Camlica Erdem Hastanesi, Uskudar, 34692 Istanbul, Turkey; e-mail: erkancure@yahoo.com

Received: 22 March 2018

Accepted: 12 November 2018

Published online: 1 April 2019

Acta Medica (Hradec Králové) 2019; 62(1): 12-18

https://doi.org/10.14712/18059694.2019.40

(c) 2019 The Authors. This is an open-access article distributed under the terms of the Creative Commons Attribution License (http://creativecommons.org/licenses/by/4.0), which permits unrestricted use, distribution, and reproduction in any medium, provided the original author and source are credited. 


\section{INTRODUCTION}

Fibromyalgia syndrome (FMS) is an extra-articular rheumatological disease characterized by widespread pain and somatic symptoms. The etiology has not yet been clarified. It is manifested with fatigue, sleep disorders, migraine, depression, irritable bowel disease, panic disorder and decreased pain threshold (1). FMS is generally seen in the middle-aged female populations (2). Although the patients frequently experience musculoskeletal pain, the laboratory findings and the radiological examinations are normal (3). There are various studies reporting the role of genetic and biochemical markers and antibodies in the FMS pathogenesis. Polymorphisms of 5-hydroxytryptamine receptor $2 \mathrm{~A}(5-\mathrm{HT} 2 \mathrm{~A})$, catechol-0-methyl transferase (COMT) gene, serotonin transporter (SERT) gene, substance $P$ gene, and cytokines genes (Interleukin [IL]-1 and IL-4) are defined in FMS. All these gene polymorphisms detected in FMS are associated with monoamine metabolism and transportation. Thus, the products of these genes are severely affected by stress (4-6).

FMS has recently been associated with the deterioration of the oxidative stress and the antioxidant balance. According to the studies, it has been observed that total antioxidant capacity and compounds of the antioxidant defense system are lower in FMS patients when compared to healthy individuals (7). It has also been reported that lipid peroxidation and the oxidative stress increase in FMS. Reactive oxygen specimens (ROS) production is considerably higher in FMS patients as a result of the oxidative stress compared to controls. Increased ROS and decreased coenzyme Q10 levels in FMS are the indicators of mitochondrial dysfunction $(8,9)$.

Thiols may react with free radicals in order to preserve the organism against tissue and cell injury which can occur due to ROS. Thiols are the compounds that contain sulfhydryl (SH) groups (10). The highest thiol amount in blood plasma exists in albumin and others proteins (11). Thiol groups form disulfide bonds as a result of being reduced by oxidant molecules (12). These structures can turn into thiols again. When the thiol and thiol/disulfide balance is deteriorated, ROS deteriorate the protein structures and cause cellular and tissue damage (13). Erel et al. developed an automated method which can separately measure thiol/ disulfide group and the evaluated thiol/disulfide rates significantly change in various diseases (10-14).

In this study, the relationship between the thiol/disulfide balance and the FMS was examined. Furthermore, we also controlled whether or not the thiol/disulfide balance is deteriorated in FMS patients when compared to healthy controls.

\section{METHODS}

This cross-sectional study was conducted in our Rheumatology, Physical Medicine and Rehabilitation clinics between January 2016 and April 2016. Eighty female patients who had complaints at least for the last three months and 64 healthy female controls were included in the study. The control group was selected among the voluntary individuals. Patient and control groups were compatible interns of age and body mass index (BMI). Patients were selected according to the ACR 2010 diagnosis criteria (15). Disease activities of FMS patients were evaluated by using Fibromyalgia Impact Questionnaire (FIQ) scores and the sleep quality of patients was assessed by using the Pittsburg sleep quality scale (PSQS). Patients and controls with concomitant systemic situations and additional properties such as vegetarian or vegan, smoking, alcohol use, pregnancy, and breastfeeding were excluded from the study. Furthermore, patients with comorbidities such as hypertension, cardiovascular disease, acute or chronic kidney disease, infections, chronic obstructive pulmonary disease, and malignancies were not included in the study. In addition, individuals who were on any type of medication (including contraceptives) or antioxidants were not included in the control group. Patients who were on drugs other than the ones used for the FMS treatment were not included in the patient group. All of the individuals were informed about the study and their written consents were obtained. The local ethics committee approval was obtained for the study.

\section{FIBROMYALGIA IMPACT QUESTIONNAIRE}

FIQ was developed by Burckhard et al. in order to evaluate the functional status of the FMS. The Turkish reliability and validity study of the scale was conducted by Sarmer et al. The physical sufficiency was evaluated via the survey which was composed of 11 items related to the daily activities $(16,17)$. The questions were aiming to highlight well-being and the loss of daily work, difficulty in working, pain, fatigue, and well-being in the morning after waking up, stiffness, anxiety, and depression. High scores presented functional limitations.

\section{PITTSBURGH SLEEPING QUALITY SCALE}

Buysse et al. developed the PSQS. The PSQS scale mainly evaluates the sleep quality, the amount of sleep, and sleep disturbances (18). The Turkish validity and reliability study of this study was conducted by Agargun et al. (19). The scale is composed of 19 questions and it is scored between 0 and 3. PSQS is composed of seven sub-dimensions such as subjective sleep quality, sleep latency, sleep duration, sleep adequacy, sleep disturbances, the use of sleeping pills, and days of dysfunction. High scores reflect the low sleep quality (18).

\section{BIOCHEMICAL ANALYSIS}

Venous blood samples of all individuals were collected into a dry tube after 10-12 hours of fasting and after $30 \mathrm{~min}-$ utes of rest. All the blood samples were collected between 8 am and 9 am under the same conditions. Blood samples were centrifuged at $1000 \mathrm{~g}$ for 15 minutes right after they were taken. Serum samples were separated into different tubes and they were stored at $-80^{\circ} \mathrm{C}$ for the analyses. Complete blood count examined via flow cytometry technique (Mindray BC-6800 Auto Hematology Analyzer, Shenzhen, China); C-reactive protein (CRP), ALT and creatinine tests 
were performed by spectrophotometric method (Abbot-Architect c8000, Japan); and erythrocyte sedimentation rate (ESR) was measured by Westergren method (Berkhum SDM-100, Turkey). Analyses were performed in the biochemistry laboratory of the Malatya State Hospital.

\section{THIOL/DISULFIDE MEASUREMENT}

Serum native thiol (NT), total thiol (TT) and disulfide levels $(\mu \mathrm{mol} / \mathrm{L})$ were measured by the method which was developed by Erel et al. (14) Disulfide/native thiol (DNT), disulfide/total thiol (DTT) and native thiol/total thiol (NTT) rates were calculated (\%). In the presence of the sodium borohydride, disulfide bonds were reduced to functional thiol groups particularly. Then, the sodium borohydride was removed with the help of formaldehyde. Reduced and NT groups were measured after the 5,5'-dithiobis- (2-nitrobenzoic) acid (DTNB) reaction. (Disulfide amount was calculated by dividing the difference between TT and NT by two.

\section{STATISTICAL ANALYSIS}

The results were given as mean $\pm \mathrm{SD}$ and median (range). SPSS program (version 18, IBM, Chicago, IL, USA) was used for the statistical analysis. The homogeneity of the distribution of the groups was evaluated by Kolmogorov Smirnov test. Student's t-test was used for the data with normal distribution, the non-homogenous data such as ESR, CRP and pain duration were analyzed with Mann Whitney-U test. Categorical data were evaluated by chi-square test. Pearson correlation test was used for correlation analysis. A $p$ value of $<0.05$ was considered significant.

\section{RESULTS}

FIQ scores, number of sensitive points, and duration of pain were higher in FMS patients when compared to the controls $(p<0.001)$. In Table 1 , sociodemographic characteristics, working status, medications, and clinical findings are given.

NT $(p=0.005)$ and NTT $(p<0.001)$ values of FMS patients were higher when compared to healthy controls. Similarly, disulfide $(\mathrm{p}<0.001)$, DNT $(\mathrm{p}<0.001)$ and DTT $(p<0.001)$ values were also higher in FMS patients compared to the controls. CRP and ESR values of FMS patients were found similar to the control group values. Thiol, disulfide and other biochemical findings of FMS patients are

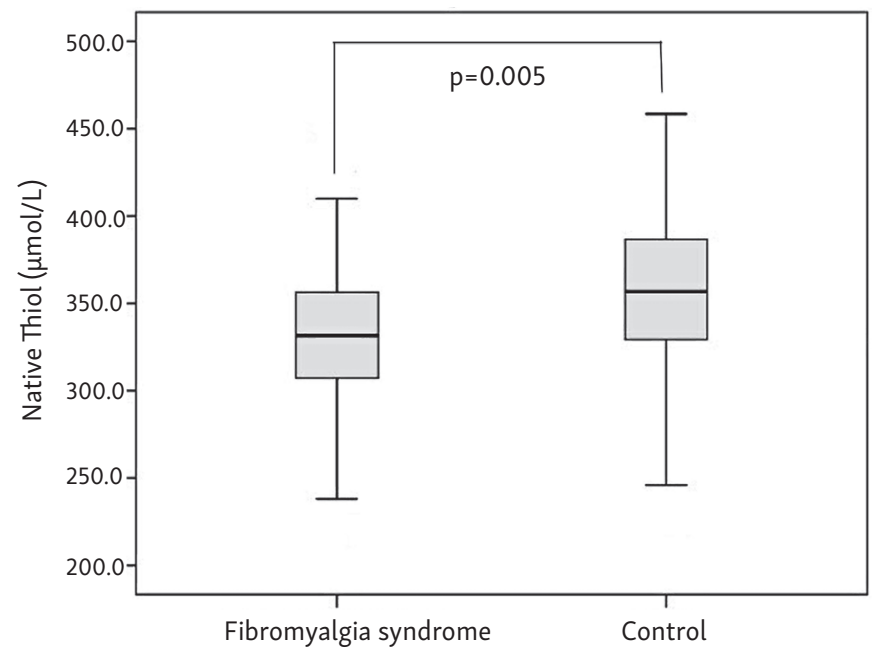

Fig. 1 Native thiol in Fibromyalgia syndrome.

Tab. 1 Demographic and clinic features of patients and control group.

\begin{tabular}{|c|c|c|c|}
\hline & Fibromyalgia $(n=80)($ mean \pm SD $)$ & Control $(n=64)($ mean \pm SD $)$ & P value \\
\hline Age (years) & $41.4 \pm 7.6$ & $39.9 \pm 10.9$ & 0.341 \\
\hline $\mathrm{BMI}\left(\mathrm{kg} / \mathrm{m}^{2}\right)$ & $26.7 \pm 3.7$ & $26.5 \pm 5.6$ & 0.811 \\
\hline Occupation (housewife/working) (n) & $46 / 34$ & $36 / 28$ & 0.234 \\
\hline Pain duration (years) (median [range]) & $3.0(0.5-20.0)$ & $0.4(0-9.0)$ & 0.001 \\
\hline FIQ score & $68.6 \pm 11.1$ & $36.2 \pm 23.7$ & 0.001 \\
\hline Number of sensitive points ( $\mathrm{n}$ ) & $13.7 \pm 2.8$ & $1.0 \pm 1.4$ & 0.001 \\
\hline PSQS & $7.5 \pm 3.7$ & $3.8 \pm 3.2$ & 0.001 \\
\hline SSRI (n) & 33 & 0 & 0.001 \\
\hline MAOIs (n) & 5 & 0 & 0.001 \\
\hline Pregabalin (n) & 24 & 0 & 0.001 \\
\hline Gabapentin (n) & 5 & 0 & 0.001 \\
\hline NSAIDs without other drugs (n) & 13 & 0 & 0.001 \\
\hline NSAIDs with other drugs (n) & 37 & 0 & 0.001 \\
\hline Opioids (n) & 0 & 0 & 1.000 \\
\hline Cannabinoids (n) & 0 & 0 & 1.000 \\
\hline Contraceptives (n) & 0 & 0 & 1.000 \\
\hline
\end{tabular}

Abbreviations: BMI - body mass index; FIQ - Fibromyalgia Impact Questionnaire; PSQS - Pittsburgh Sleeping Quality Scale; SSRI - selective serotonin reuptake inhibitor; MAOIs - monoamine oxidase inhibitors; NSAIDs - non-steroidal anti-inflammatory drugs. 
Tab. 2 Results of thiol, disulfide and biochemical parameters in fibromyalgia and control groups.

\begin{tabular}{|l|l|l|l|}
\hline & Fibromyalgia $(\mathbf{n}=\mathbf{8}$ ) $)$ (mean \pm SD) & Control $(\mathbf{n}=\mathbf{6 4})$ (mean \pm SD) & P value \\
\hline Native Thiol $(\mu \mathrm{mol} / \mathrm{L})$ & $330.6 \pm 46.1$ & $356.8 \pm 55.5$ & 0.005 \\
\hline Total Thiol $(\mu \mathrm{mol} / \mathrm{L})$ & $369.5 \pm 49.0$ & $381.3 \pm 51.3$ & 0.183 \\
\hline Native thiol/total thiol $\times 100$ & $89.4 \pm 3.2$ & $93.3 \pm 4.0$ & 0.001 \\
\hline Disulfide $(\mu \mathrm{mol} / \mathrm{L})$ & $19.4 \pm 6.3$ & $12.2 \pm 6.3$ & 0.001 \\
\hline Disulfide/native thiol $\times 100$ & $5.9 \pm 2.0$ & $3.6 \pm 2.5$ & 0.001 \\
\hline Disulfide/total thiol $\times 100$ & $5.2 \pm 1.6$ & $3.3 \pm 2.0$ & 0.001 \\
\hline BUN $(\mathrm{mg} / \mathrm{dl})$ & $12.9 \pm 1.2$ & $13.3 \pm 1.1$ & 0.062 \\
\hline Creatinine $(\mathrm{mg} / \mathrm{dl})$ & $0.7 \pm 0.4$ & $0.7 \pm 0.1$ & 0.454 \\
\hline AST $(\mathrm{IU} / \mathrm{l})$ & $17.9 \pm 6.4$ & $19.5 \pm 8.0$ & 0.201 \\
\hline ALT $(\mathrm{IU} / \mathrm{l})$ & $17.4 \pm 10.5$ & $19.4 \pm 9.7$ & 0.237 \\
\hline HB $(\mathrm{g} / \mathrm{dl})$ & $12.9 \pm 1.2$ & $13.3 \pm 1.1$ & 0.062 \\
\hline CRP $(\mathrm{mg} / \mathrm{dl})$ (median [range]) & $0.3(0-14.0)$ & $0.3(0-4.3)$ & 0.480 \\
\hline ESR $(\mathrm{mm} / \mathrm{h})$ & $15.1 \pm 8.3$ & $15.5 \pm 9.3$ & 0.817 \\
\hline
\end{tabular}

Abbreviations: BUN - blood urea nitrogen; AST - aspartate aminotransferase; ALT - alanine aminotransferase; CRP - C-reactive protein; ESR - erythrocyte sedimentation rate.

given in Table 2. The NT levels are shown in Figure 1 and disulfide levels are shown in Figure 2.

There was a positive correlation between FIQ and BMI $(\mathrm{r}=0.227, \mathrm{p}=0.006)$ and age $(\mathrm{r}=0.240, \mathrm{p}=0.004)$. In our study, there was not any correlation between CRP, ESR and FIQ, number of sensitive points, and duration of pain, thiol, and disulfide levels. There was a negative correlation between FIQ scores and NTT levels. We found a positive relationship between FIQ scores and disulfide, DNT and DTT values. There was a negative correlation between the number of sensitive points and NT and NTT levels while a positive correlation between D, DNT, and DTT was found. There was a positive relationship between the pain duration and disulfide values. FIQ scores, number of sensitive points and pain duration and thiol and disulfide levels had positive correlations. Results are given in Table 3. Correlation between disulfide and FIQ score is shown in Figure 3. Additionally, there was a negative correlation

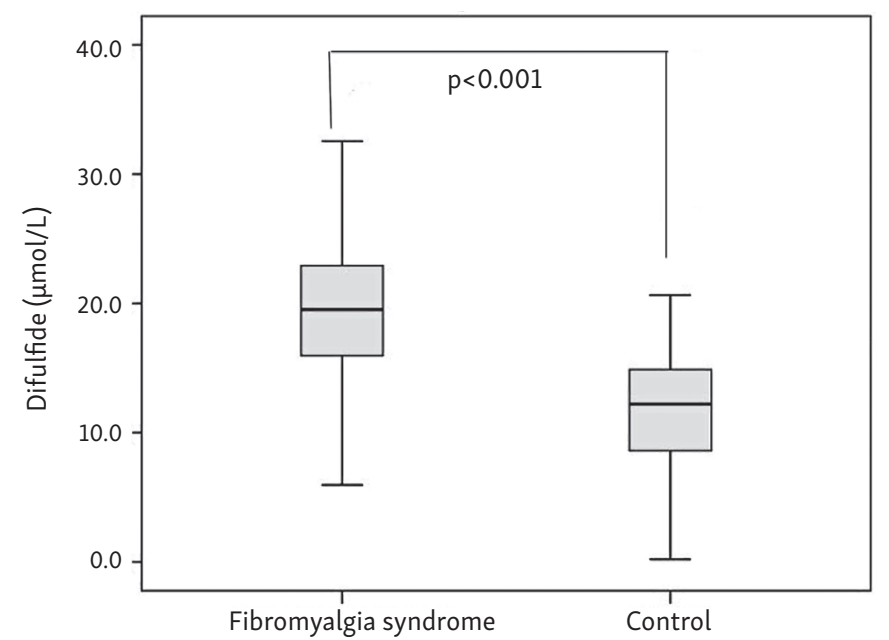

Fig. 2 Disulfide levels in Fibromyalgia syndrome. between PSQS and NT $(r=-0.211, p=0.012), T T(r=-0.170$, $\mathrm{p}=0.043)$ and NTT $(\mathrm{r}=-0.190, \mathrm{p}=0.023)$. However, we found a positive correlation between PSQS scores and disulfide $(\mathrm{r}=0.166, \mathrm{p}=0.048)$, DNT $(\mathrm{r}=0.172, \mathrm{p}=0.040)$ and DTT $(r=0.193, p=0.021)$.

\section{DISCUSSION}

The thiol levels of FMS patients were lower and the disulfide levels of patients were higher compared to control individuals. There was a significant relationship between thiol levels and FIQ scores, the number of sensitive points, and PSQS scores. FMS is commonly observed in women and it is characterized by a widespread pain. However, the etiology of FMS has not yet been clarified. FMS is associated with increased oxidative stress, widespread pain, fatigue and depression $(20,21)$. Chronic inflammation and

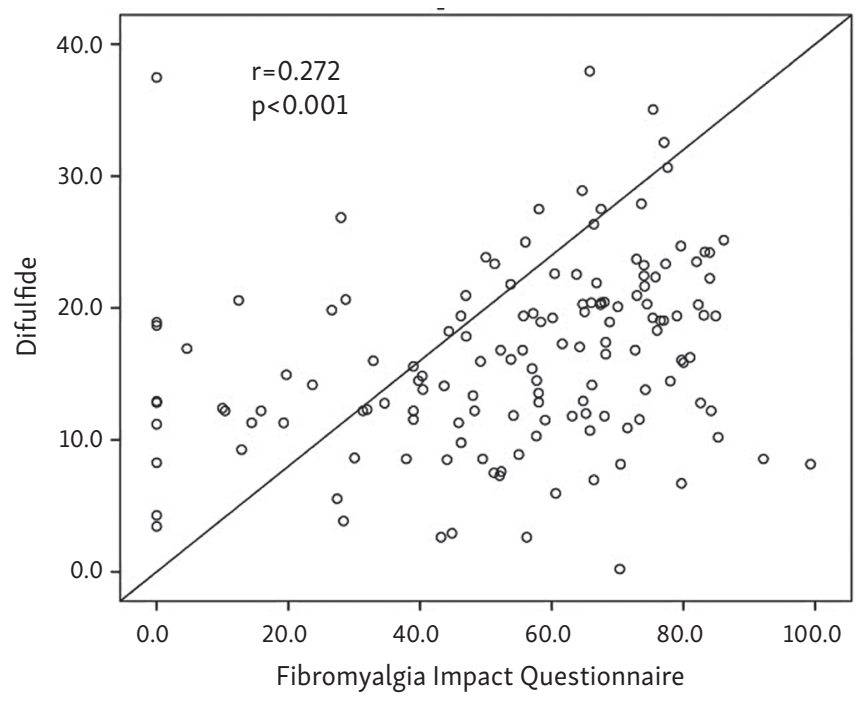

Fig. 3 Correlation between disulfide and FIQ score. 
Tab. 3 Results of thiol, disulfide and other parameters for correlation analysis in fibromyalgia.

\begin{tabular}{|c|c|c|c|c|c|c|}
\hline & \multicolumn{2}{|c|}{ FIQ score } & \multicolumn{2}{|c|}{ Number of sensitive points } & \multicolumn{2}{|c|}{ Pain duration } \\
\hline & r value & p value & r value & $p$ value & r value & p value \\
\hline Native Thiol & -0.142 & 0.091 & -0.267 & 0.001 & -0.080 & 0.341 \\
\hline Total Thiol & -0.068 & 0.421 & -0.133 & 0.113 & -0.024 & 0.778 \\
\hline Native Thiol/Total Thiol & -0.240 & 0.004 & -0.476 & 0.001 & -0.164 & 0.051 \\
\hline Disulfide & 0.272 & 0.001 & 0.492 & 0.001 & 0.204 & 0.015 \\
\hline Disulfide/Native Thiol & 0.220 & 0.008 & 0.451 & 0.001 & 0.149 & 0.075 \\
\hline Disulfide/Total Thiol & 0.240 & 0.004 & 0.475 & 0.001 & 0.164 & 0.050 \\
\hline Number of sensitive points & 0.684 & 0.001 & & & 0.417 & 0.001 \\
\hline Pain Duration & 0.396 & 0.001 & 0.417 & 0.001 & & \\
\hline
\end{tabular}

Abbreviations: FIQ, Fibromyalgia Impact Questionnaire.

oxidative stress can cause deterioration in the hypothalamic-pituitary-adrenal axis and thus FMS can occur (22). Oxidative stress can lead to mitochondrial damage and the deterioration of the energy balance and antioxidant defense system by affecting the protein kinases. Mitochondrial damage also takes place in FMS etiopathogenesis (23, 24). In the study of Neval et al., it was shown that total oxidant status and oxidative stress status were higher and total antioxidant status was lower in FMS patients when compared to the controls (25). The study by Fatima et al. showed that lipid peroxidation levels of FMS patients were higher and levels of antioxidant defense system enzymes such as glutathione peroxidase (GPx) and glutathione reductase in FMS patients were lower than the controls (21). However, there are studies stating that oxidant and antioxidant capacity of FMS patients were similar with the control group. Akbas et al. showed that GPx enzyme levels were similar in both FMS patients and healthy individuals $(26,27)$. Similarly, Toker et al. indicated that the ischemia-modified albumin levels were also similar in FMS patients and controls (26). Bozkurt et al. found that the oxidant status and oxidative stress index values of FMS patients were higher than controls but total antioxidant status and paraoxonase-1 levels were similar in patients and controls (28). Regarding these results, there are contradictory findings in the literature.

Glutathione is a tripeptide containing thiol and it protects the organism from the oxidative stress by activity of the enzyme GPx (29). In many studies, instead of studying the direct thiol group, glutathione and GPx levels were studied. Many drugs have a positive or negative effect on thiol levels (30). Our patients used selective serotonin reuptake inhibitors (SSRIs), monoamine oxidase inhibitors (MAOIs), non-steroidal anti-inflammatory drugs (NSAIDs), pregabalin and gabapentin. SSRIs are found to increase GPx, glutathione and thiol levels in the brain tissue while they cause a severe decrease in GPx, glutathione and thiol levels in the liver tissue (31). It has been reported that pregabalin, gabapentin, and NSAIDs decrease GPx levels (30-33). The low level of GPx and glutathione indirectly indicates the low thiol levels. In this study, low thiol levels in FMS patients may be due to the use of these drugs.

It was shown by Sarifakioglu et al. that there was a strong relationship between the oxidative stress parameters and FIQ, Beck depression inventory and visual analog scale scores (34). In our study, there was a strong positive relationship between thiol levels of FMS patients and their FIQ scores, number of sensitive points and the PSQS. On the other hand, we found a negative correlation between disulfide levels and FIQ scores, sensitive points, and the PSQS. Thiols have roles in the maintenance of the oxidative balance and they are molecules that contain sulfhydryl groups (10). Thiols create disulfide bonds in the presence of oxygen via oxidation (12). Thiols are mainly present in albumin and other protein derives such as cysteine, methionine, and reduced glutathione (35). Thiols have important roles in the clearance of hypochlorous acid from the body (36). It shows that oxidative stress increases due to the low thiol levels and high disulfide levels. The plasma thiol measurement was previously performed by using old manual methods (37). However, Erel et al. have recently found a cheap, easy, reliable, and fully automated method and the thiol/disulfide balance can be measured by using this technique (14). Using this method revealed that the balance of thiol/disulfide was deteriorated in favor of the disulfide in diseases such as Familial Mediterranean fever, cardiac disease and diabetes mellitus which are characterized with an enhanced oxidative stress (13, 38,39 ). In our study, we showed that FMS patients have lower thiol levels and higher disulfide levels compared to controls. In FMS patients, there was a strong relationship between the number of sensitive points and the thiol/disulfide levels. Furthermore, the sleeping status and the thiol levels of the patients had a relationship. Our findings lead us to think that thiol/disulfide balance is deteriorated in FMS and oxidative stress has a role in the etiology of this disease.

In our study, CRP levels of our patients were not high and there was no correlation between the CRP levels and thiol levels of patients. CRP is a good indicator of inflammation. However, fibromyalgia is not an inflammation-related disease and CRP increase is not expected in the course (3). Xiao et al. showed that high sensitive (hs)-CRP levels were higher in FMS patients when compared to controls (40). However, patient and control groups were not matched regarding BMI-matched; FMS patients and controls had prominent weight difference. The group found that hs-CRP increased even though the 
inflammation status was low. Rus et al. showed that both normal and the over-weighted FMS patients had higher CRP levels compared to the controls. However, a relationship was present between CRP levels and disease activity of the over-weighted FMS patients (41). Rus et al. had a smaller sample size and it is possible that the results might be affected due to the sample size. Xiao et al. and Rus et al. reported high CRP levels in obese FMS patients. It is well known that obesity causes inflammation. Therefore, studies of Xiao et al. and Rus et al. may found high CRP levels in FMS patients due to the obesity status of individuals rather than the disease. CRP is mostly secreted from the liver and CRP release is mainly induced by interleukin-6 (IL)- 6 . If the IL- 6 release is low or if there is a lack of response against IL-6, CRP levels may not increase (42). It is known that IL-6 levels are not increased in FMS and both patients and the controls have similar levels of IL- 6 cytokine (43, 44). In our study, we found similar levels of CRP in both the patient group and the control group most probably due to the lack of increase in IL-6 levels. Furthermore, increased cytokine level increases the oxidative stress but cytokine release is not required for the formation of the oxidative stress (45). This can be the reason why we could not reveal any relationship between CRP levels and thiol groups.

As a result, this study concluded that thiol levels were lower and disulfide levels were higher in FMS patients when compared to the controls. The change in the thiol/ disulfide balance in favor of disulfide amounts reflects the oxidative stress. Increased oxidative stress can have a role in the deterioration of the thiol balance in FMS patients. It is possible to examine whether or not drugs with thiol groups have positive effects on FMS patients.

\section{REFERENCES}

1. Bennett RM, Jones J, Turk DC, Russell IJ, Matallana L. An internet survey of 2,596 people with fibromyalgia. BMC Musculoskelet Disord 2007; 8: 27.

2. Couto CI, Natour J, Carvalho AB. Fibromyalgia: its prevalence and impact on the quality of life on a hemodialyzed population. Hemodial Int 2008; 12: 66-72.

3. Arnold LM, Clauw DJ, McCarberg BH. FibroCollaborative. Improving the recognition and diagnosis of fibromyalgia. Mayo Clin Proc 2011; 86: 457-64.

4. Cohen H, Neumann L, Glazer Y, Ebstein RP, Buskila D. The relationship between a common catechol-O-methyltransferase (COMT) polymorphism val (158) met and fibromyalgia. Clin Exp Rheumatol 2009; 27: 51-6.

5. Wallace DJ, Linker-Israeli M, Hallegua D, Silverman S, Silver D, Weisman MH. Cytokines play an aetiopathogenetic role in fibromyalgia: a hypothesis and pilot study. Rheumatology (Oxford) 2001; 40: 743-9.

6. Kato K, Sullivan PF, Evengard B, Pedersen NL. Importance of genetic influences on chronic widespread pain. Arthritis Rheum 2006; 54: 1682-6.

7. Ozgocmen S, Ozyurt H, Sogut S, Akyol O. Current concepts in the pathophysiology of fibromyalgia: the potential role of oxidative stress and nitric oxide. Rheumatol Int 2006; 26: 585-97.

8. Altindag $\mathrm{O}$, Celik $\mathrm{H}$. Total antioxidant capacity and the severity of the pain in patients with fibromyalgia. Redox Rep 2006; 11: 131-5.

9. Cordero MD, de Miguel M, Carmona-Lopez I, Bonal P, Campa F, Moreno-Fernandez AM. Oxidative stress and mitochondrial dysfunction in fibromyalgia. Neuro Endocrinol Lett 2010; 31: 169-73.

10. Altiparmak IH, Erkus ME, Sezen H, et al. Evaluation of thiol levels, thiol/disulfide homeostasis and their relation with inflammation in cardiac syndrome X. Coron Artery Dis 2016; 27: 295-301.

11. Jovanovic VB, Pavicevic ID, Takic MM, Penezic-Romanjuk AZ, Acimovic JM, Mandic LM. The influence of fatty acids on determination of human serum albumin thiol group. Anal Biochem 2014;448: 50-7.
12. Yuksel M, Ates I, Kaplan M, et al. The dynamic thiol/disulphide homeostasis in inflammatory bowel disease and its relation with disease activity and pathogenesis. Int J Colorectal Dis 2016; 31: 122931.

13. Kundi H, Ates I, Kiziltunc E, et al. A novel oxidative stress marker in acute myocardial infarction; thiol/disulphide homeostasis. Am J Emerg Med 2015; 33: 1567-71.

14. Erel O, Neselioglu S. A novel and automated assay for thiol/disulphide homeostasis. Clin Biochem 2014; 47: 326-32.

15. Wolfe F, Clauw DJ, Fitzcharles MA, et al. The American College of Rheumatology preliminary diagnostic criteria for fibromyalgia and measurement of symptom severity. Arthritis Care Res (Hoboken) 2010; 62: 600-10.

16. Burckhardt CS, Clark SR, Bennett RM. The fibromyalgia impact questionnaire: development and validation. J Rheumatol 1991; 18: 728-33.

17. Sarmer S, Ergin S, Yavuzer G. The validity and reliability of the Turkish version of the Fibromyalgia Impact Questionnaire. Rheumatol Int 2000; 20: 9-12.

18. Buysse DJ, Reynolds CF 3rd, Monk TH, Berman SR, Kupfer DJ. The Pittsburgh Sleep Quality Index: a new instrument for psychiatric practice and research. Psychiatry Res 1989; 28: 193-213.

19. Ağargün MY, Kara H, Anlar Ö. Pittsburgh Uyku Kalitesi İndeksiınin geçerliği ve güvenirliği. Turk Psikiyatr Derg 1996; 7: 107-15.

20. Peres Klein C, Rodrigues Cintra M, Binda N, et al. Coadministration of Resveratrol and Rice Oil Mitigates Nociception and Oxidative State in a Mouse Fibromyalgia-Like Model. Pain Res Treat 2016; 2016: 3191638.

21. Fatima G, Das SK, Mahdi AA. Some oxidative and antioxidative parameters and their relationship with clinical symptoms in women with fibromyalgia syndrome. Int J Rheum 2017; 20: 39-45.

22. Romano GF, Tomassi S, Russell A, Mondelli V, Pariante CM. Fibromyalgia and chronic fatigue: the underlying biology and related theoretical issues. Adv Psychosom Med 2015; 34: 61-77.

23. Alcocer-Gomez E, Garrido-Maraver J, Bullon P, et al. Metformin and caloric restriction induce an AMPK-dependent restoration of mitochondrial dysfunction in fibroblasts from Fibromyalgia patients. Biochim Biophys Acta 2015; 1852: 1257-67.

24. Sanchez-Dominguez B, Bullon P, Roman-Malo L, et al. Oxidative stress, mitochondrial dysfunction and, inflammation common events in skin of patients with Fibromyalgia. Mitochondrion 2015; 21:69-75.

25. Neyal M, Yimenicioglu F, Aydeniz A, et al. Plasma nitrite levels, total antioxidant status, total oxidant status, and oxidative stress index in patients with tension-type headache and fibromyalgia. Clin Neurol Neurosurg 2013; 115: 736-40.

26. Toker A, Kucuksen S, Kucuk A, Cicekler H. Serum ischemia-modified albumin and malondialdehyde levels and superoxide dismutase activity in patients with fibromyalgia. Clin Lab 2014; 60: 1609-15.

27. Akbas A, Inanir A, Benli I, Onder Y, Aydogan L.Evaluation of some antioxidant enzyme activities (SOD and GPX) and their polymorphisms (MnSOD2 Ala9Val, GPX1 Pro198Leu) in fibromyalgia. Eur Rev Med Pharmacol Sci 2014; 18: 1199-203.

28. Bozkurt M, Caglayan M, Oktayoglu P, et al. Serum prolidase enzyme activity and oxidative status in patients with fibromyalgia. Redox Rep 2014; 19: 148-53.

29. Dickinson DA, Forman HJ. Cellular glutathione and thiols metabolism. Biochem Pharmacol 2002; 64: 1019-26.

30. Wolf WA, Kuhn DM. Role of essential sulfhydryl groups in drug interactions at the neuronal 5-HT transporter. Differences between amphetamines and 5-HT uptake inhibitors. J Biol Chem 1992; 267: 20820-5.

31. Abdel Salam OM, Mohammed NA, Sleem AA, Farrag AR. The effect of antidepressant drugs on thioacetamide-induced oxidative stress. Eur Rev Med Pharmacol Sci 2013; 17: 735-44.

32. Erdemir F, Atilgan D, Firat F, Markoc F, Parlaktas BS, Sogut E. The effect of sertraline, paroxetine, fluoxetine and escitalopram on testicular tissue and oxidative stress parameters in rats. Int Braz J Urol 2014; 40: 100-8.

33. Abdel-Salam OM, Khadrawy YA, Mohammed NA, Youness ER. The effect of gabapentin on oxidative stress in a model of toxic demyelination in rat brain. J Basic Clin Physiol Pharmacol 2012; 23: 61-8.

34. Sarıfakıoğlu B, Güzelant AY, Güzel EC, Güzel S, Kızıler AR. Effects of 12 -week combined exercise therapy on oxidative stress in female fibromyalgia patients. Rheumatol Int 2014; 34: 1361-7.

35. Turell L, Radi R, Alvarez B. The thiol pool in human plasma: the central contribution of albumin to redox processes. Free Radic Biol Med 2013; 65: 244-53.

36. Storkey C, Davies MJ, Pattison DI. Reevaluation of the rate constants for the reaction of hypochlorous acid ( $\mathrm{HOCl})$ with cysteine, methi- 
onine, and peptide derivatives using a new competition kinetic approach. Free Radic Biol Med 2014; 73: 60-6.

37. Ellman GL, Lysko H. Disulfide and sulfhydryl compounds in TCA extracts of human blood and plasma. J Lab Clin Med 1967; 70: 518-27.

38. Ates I, Kaplan M, Yuksel M, et al. Determination of thiol/disulphide homeostasis in type 1 diabetes mellitus and the factors associated with thiol oxidation. Endocrine 2016; 51:47-51.

39. Yucel A, Sanhal CY, Daglar K, Kara O, Uygur D, Erel O. Thiol/disulphide homeostasis in pregnant women with Familial Mediterranean fever. Redox Rep 2016; 21: 287-91.

40. Xiao Y, Haynes WL, Michalek JE, Russell IJ. Elevated serum high-sensitivity C-reactive protein levels in fibromyalgia syndrome patients correlate with body mass index, interleukin- 6 , interleukin- 8 , erythrocyte sedimentation rate. Rheumatol Int 2013; 33: 1259-64.

41. Rus A, Molina F, Gasso M, Camacho MV, Peinado MA, Moral ML.
Nitric Oxide, Inflammation, Lipid Profile, and Cortisol in Normaland Overweight Women With Fibromyalgia. Biol Res Nurs 2016; 18: 138-46.

42. Puel A, Picard C, Lorrot M, et al. Recurrent staphylococcal cellulitis and subcutaneous abscesses in a child with autoantibodies against IL-6. J Immunol 2008; 180: 647-54.

43. Fatima G, Mahdi AA, Das SK, et al. Lack of Circadian Pattern of Serum TNF- $\alpha$ and IL- 6 in Patients with Fibromyalgia Syndrome. Indian J Clin Biochem 2012; 27: 340-3.

44. Ghizal F, Das SK, Verma N, Mahdi AA. Evaluating relationship in cytokines level, Fibromyalgia Impact Questionnaire and Body Mass Index in women with Fibromyalgia syndrome. J Back Musculoskelet Rehabil 2016; 29: 145-9.

45. Gebicki JM. Oxidative stress, free radicals and protein peroxides. Arch Biochem Biophys 2016; 29: 145-9. 